\title{
Reflection and Diffraction Phenomena of Blast Wave Propagation in Nuclear Fuel Cycle Facility*
}

\author{
Akihiko MIURA $^{* *}$, Akiko MATSUO ${ }^{* * *}$, Toshiharu MIZUKAKI ${ }^{* * * *}$, Takuya SHIRAISHI ${ }^{* * *}$, \\ Go UTSUNOMIYA****, Kazuyoshi TAKAYAMA**** and Ichiro NOJIRI**
}

\begin{abstract}
This paper presents the results of an optical experiment which is carried out to measure the pressure and to observe the wave propagations when an explosion occurs in a model of a nuclear facility for preventing and mitigating the serious damage of nuclear facility. Numerical simulation is also performed to compare the phenomena in a model of nuclear facility. Nuclear facility is simulated as the several closed rooms in these experiments and simulations, because the nuclear facility is composed of many closed rooms. As a result, typical tendencies of pressure history are obtained, and it is confirmed that the explosion which occurs in a closed space is reflected in the complexity at the walls and interfered mutually with progressing waves. Finally, experimental results are compared with a numerical simulation. It is confirmed that the results of a numerical simulation show a good agreement with experimental results.
\end{abstract}

Key Words: Blast Wave, Explosion, Reflection, Diffraction, Numerical Simulation, Holographic Interferometry, Pressure Measurement, Nuclear Fuel Cycle Facility, Safety

\section{Introduction}

Explosion is a phenomenon of a release of the energy from high energy materials. When an explosion occurs accidentally, serious damage of facilities or human properties can be observed. In the last decade, there have been three serious explosion accidents in nuclear facilities. We, the Japan Nuclear Cycle Development Institute (JNC, reorganized from Power Reactor and Nuclear Fuel Development Corporation), experienced a fire and explosion on March 11, 1997. Through a three year investigation, the cause of this incident is almost established ${ }^{(1)}$. In addition, judging from the explosion accidents in the DOE (Dept. of Energy) Hanford site (USA) ${ }^{(2)}$ and Tomsk-7 (Russia) ${ }^{(3)}$,

* Received 4th November, 2003 (No. 03-4152)

* Japan Nuclear Cycle Development Institute, 4-33 Muramatsu, Tokai-mura, Naka-gun, Ibaraki 319-1194, Japan. E-mail: amiura@tokai.jnc.go.jp

*** Department of Science and Technology, Keio University, 3-14-1 Hiyoshi, Kohoku-ku, Yokohama, Kanagawa, 2238522, Japan

**** Shock Wave Research Center, Institute of Fluid Science, Tohoku University, 2-1-1, Katahira, Aoba-ku, Sendai, Miyagi, 980-8577, Japan the potential hazards of an explosion in nuclear fuel cycle process are understood in a nuclear facility. Also, recent news topics said the risk of terrorism using the explosive materials to nuclear facility. Based on some risks and hazards, it is thought that the blast wave propagation generated by an explosion accident in nuclear facility and interactions between blast wave and complex media are ones of the important research topics of the safety for preventing and mitigating the serious damage of a nuclear facility.

It is thought that the explosion in nuclear fuel cycle facility is simulated in the closed space, because nuclear fuel cycle facility is constructed by strong reinforced concrete walls to prevent flowing out the radioactive materials and the radioactive damage. In order to clear the cause of these accidents and to evaluate the safety in the nuclear fuel cycle facility, phenomenon in closed space need to be understood deeply. Therefore, we have started to the visualization experiment to observe the wave propagation, pressure measurements to evaluate the pressure loading to some components and development for numerical simulation program to estimate the phenomena generated in closed space.

This paper describes the results obtained from these experiments and simulations. And wave propagation and 
pressure loading to walls are discussed to evaluate the safety for nuclear fuel cycle facility.

\section{Experiments}

\subsection{Test section and explosive system}

Explosion experiments were carried out in a test section which is made of Plexiglas for the holographic interferometry. A test section is modeled as a closed room which is called a 'cell' in the Bituminization Demonstration Facility (BDF) in JNC. BDF is one of a nuclear facility in which a fire and an explosion incident occurred actually. The size of a test section is $0.12 \times 0.74 \times 0.22 \mathrm{~m}$ (Fig. 1). In this figure, to the left side is a small room (Room A) whose size is $0.10 \times 0.10 \times 0.10 \mathrm{~m}$ and to the right side is a large room (Room B) whose size is $0.10 \times$ $0.50 \times 0.10 \mathrm{~m}$. Each room is connected by the window whose size is $0.04 \times 0.04 \mathrm{~m}$. Two rooms on the 2 nd floor $(0.10 \times 0.10 \times 0.15$ and $0.10 \times 0.10 \times 0.05 \mathrm{~m})$ are also connected by the door space $(0.05 \times 0.03 \mathrm{~m})$, and the $1 \mathrm{st}$ and 2 nd floors are connected by the roof hatch $(0.08 \times 0.03 \mathrm{~m})$.

Explosives such as $10 \mathrm{mg}$ of silver azide are exploded by laser beam for the explosion generation. For this explosive system, $120 \mathrm{~mJ}$ YAG laser and $2 \mathrm{~m}$-optical fiber were employed (in Fig. 2).

\subsection{Holographic interferometry}

Visualization technologies of explosion using the change in the refractive index by density variation have been developed to observe the phenomena of high speed wave propagation. The double exposure holographic interferometry is one of the visualization technologies which

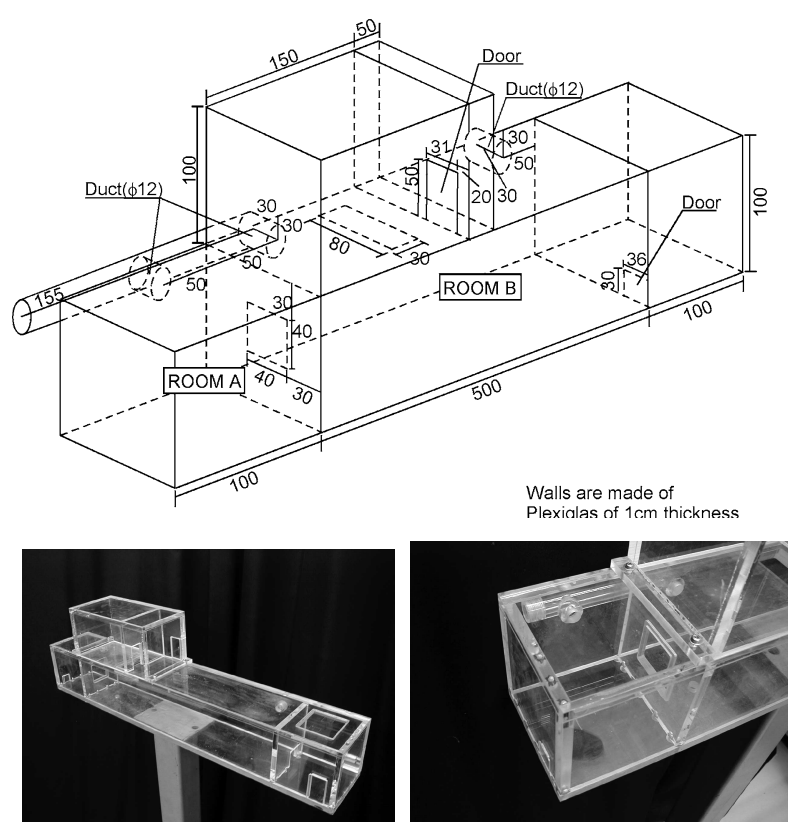

Fig. 1 Test section (scaled model of 1st and 2nd floor of bituminization demonstration facility) are suitable for this experiment, because this technology does not require such highly accurate optical devices and can take photographs of the phenomena in the large test section $^{(4)}$.

Figure 3 presents a schematic diagram of the holographic interferometric optical arrangement. A holographic ruby laser with $3.0 \mathrm{~J} /$ pulse, wavelength $694.3 \mathrm{~mm}$ and pulse duration of $25 \mathrm{~ns}$ was the light source. The ruby laser (RL) was always operated in the $\mathrm{TEM}_{00}$ mode. Often, without using double pulse operations, the RL was repeatedly operated twice at approximately $30 \mathrm{~s}$ intervals. A first exposure was carried out before the passage of the shock wave and a second exposure was triggered by the output signal of one of the pressure transducers via a delay controller when a wave arrived at a particular location

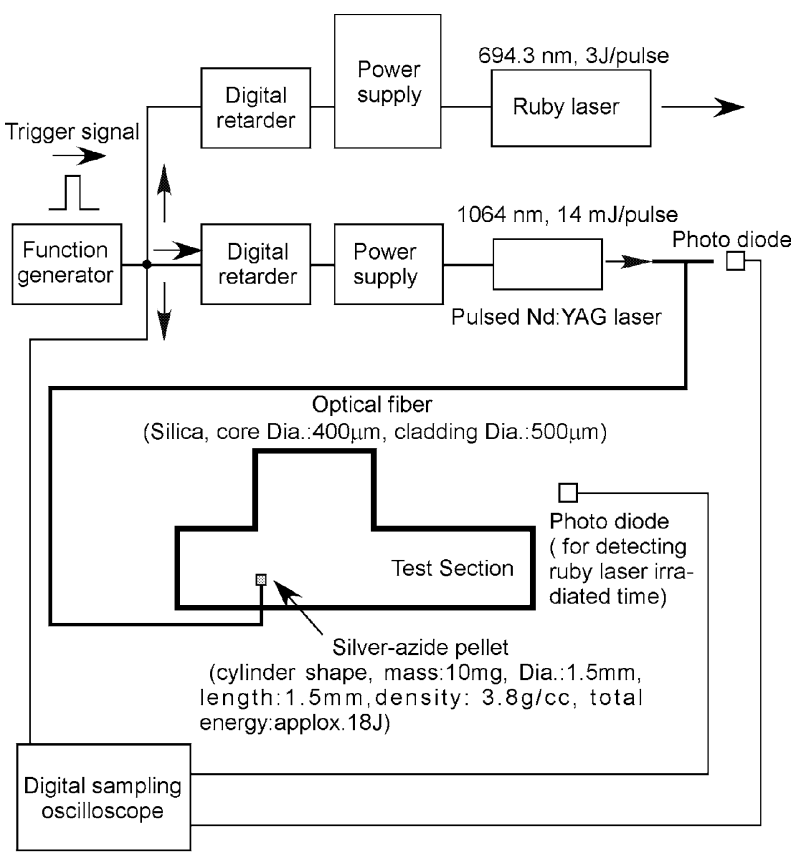

Fig. 2 Explosive system

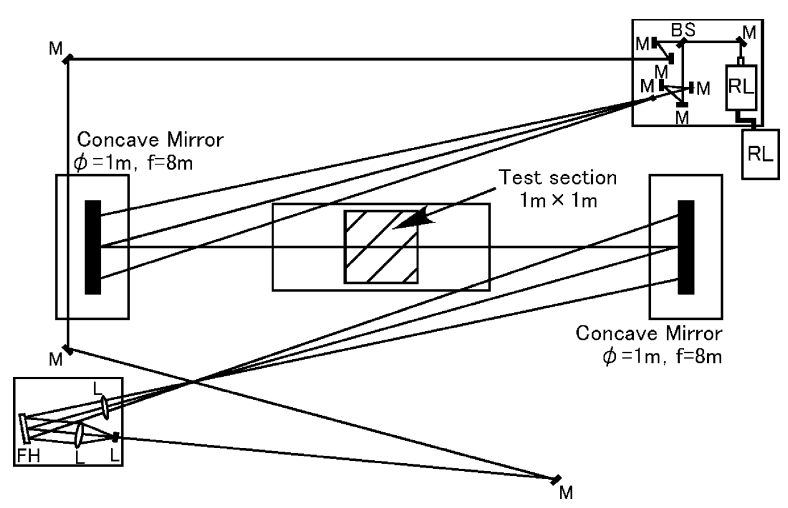

Fig. 3 Optical setup of double exposure holographic interferometry 


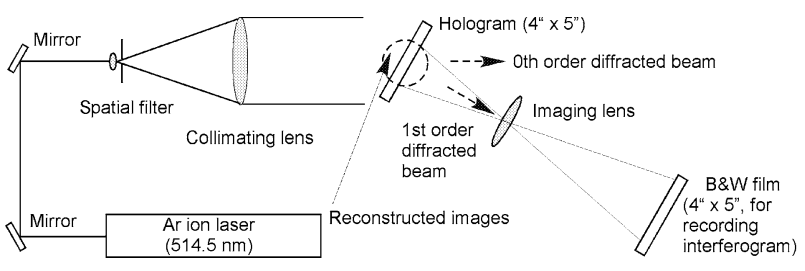

Fig. 4 Reconstruction system

in the test section.

In Fig. 3, the laser beam of RL is split into an object beam $(\mathrm{OB})$ and a reference beam (RB) using a 5:5 beam splitter (BS) and half of the ruby laser light was directed to the object beam. A pair of concave mirrors, of $1000 \mathrm{~mm}$ diameter and $8000 \mathrm{~mm}$ focal length, are used. The reference beam, which is transmitted to a film folder by concave mirrors and collimated by a plano-convex lens on the holder, was superimposed with the object beam on the holder. The difference in path lengths (approximately $25.0 \mathrm{~m}$ ) between the object and reference beams was adjusted, by using plane mirrors (M), within no more than a few centimeters. Images of the explosion, caused by a change of refractive index associated with local density variation across the explosion, were focused on holo-films by using a relay lens. ILFORD $100 \times 125 \mathrm{~mm}$ sheet films were used, which were placed on the film holder.

Figure 4 presents the reconstruction system. A He-Ne laser of $633 \mathrm{~nm}$ wavelength was used for reconstruction. In this system, the point light source became a parallel ray in the convex lens, and when the ray passed and diffracted at the exposed holo-film, the real image was obtained. The image formed in the formation lens was recorded on the film sheet.

\subsection{Pressure measurement}

Pressure inside the scaled model is measured using semiconductor pressure transducers (Kistler 603B). Kistler 603B is very sensitive to measure pressure fractuation and has very high responsibility to transport to digital signals (in Fig. 5). Transmitted signals are amplified, input the digital oscilloscope and recorded in personal computer.

Pressure is measured at seven points in scaled model (Fig. 6). These points are put on the corner of the walls and faces of walls.

\subsection{Experimental condition}

The case of explosion in room $\mathrm{A}$ and the case of room $\mathrm{B}$ are considered. In the case of explosion in room $\mathrm{A}$ is conducted in small room whose doors, windows and hatches are closed. In the contrariety, in the case of explosion in room $\mathrm{B}$, all doors, windows and hatches are opened.

In order to minimize the change in ambient conditions, any visualization experiments and pressure measurements are carried out within 24 hours when

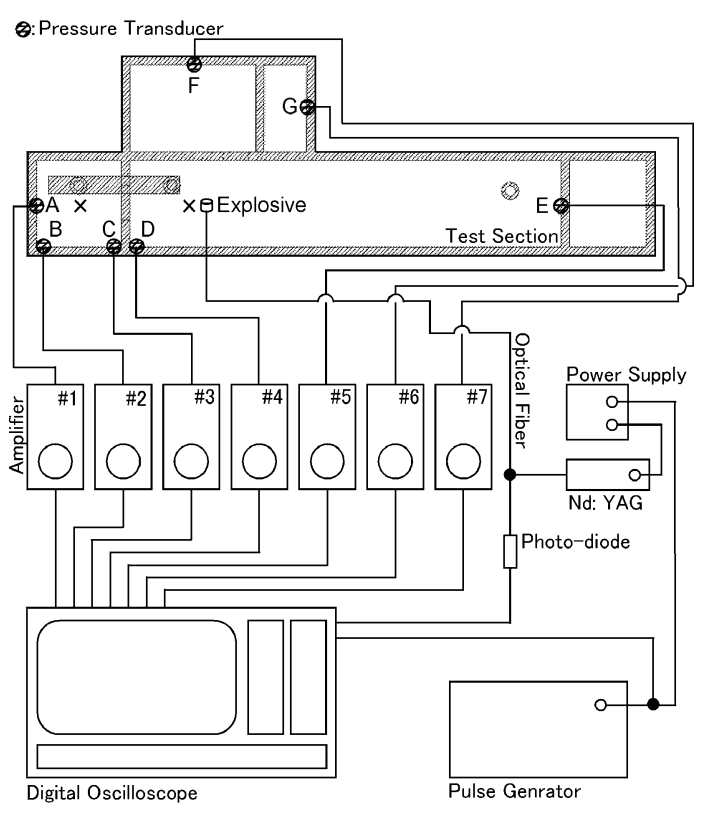

Fig. 5 Diagram of pressure measurement
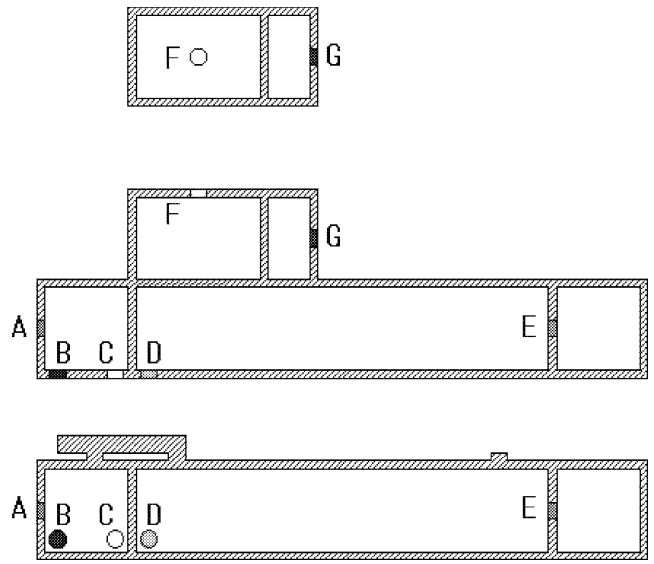

Fig. 6 Points of pressure measurement

the appropriate weather conditions existed in December, 2001. Sound velocity generated by explosives was estimated about $334-338 \mathrm{~m} / \mathrm{s}$, when the air temperature was almost 5-10 centi-degrees.

\section{Experimental Results}

Holographic photographs of time dependence are shown in Figs. 7 and 8. Figure 7 shows interference fringe which is obtained by the case of explosion generated in room A and Fig. 8 is observed the case of explosion generated in room B. All holographs were taken $0.02-0.03 \mathrm{~ms}$ intervals.

In the case of explosion in room $\mathrm{A}$, at $0.050 \mathrm{msec}$ after explosion, propagating waves reached nearest walls and Mach or regular reflection occurs here. And reflected waves are overlapped with progressing wave complexly 
after $0.108 \mathrm{~ms}$.

In the case of explosion in room B, we can also observe the wave reflection and it causes the interference. Reflected wave and the progressing wave cause interference and they pass the window which is installed between room A and room B. It is found that passed wave and the wave that is reflected on the wall in the vertical direction are caused interference. And second shock wave occurred in $0.200 \mathrm{~ms}$ after. It was possible to load high pressure at the corner of the room $\mathrm{B}$ repeatedly.

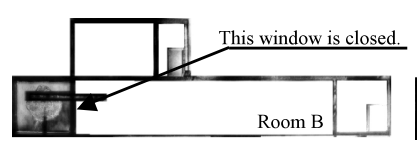

(a) $0.031 \mathrm{~ms}$

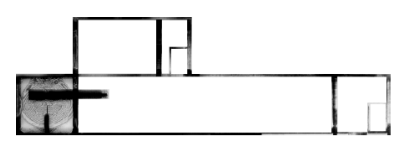

(c) $0.080 \mathrm{~ms}$

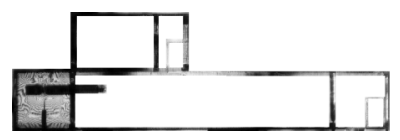

(e) $0.135 \mathrm{~ms}$

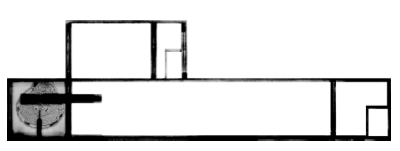

(b) $0.059 \mathrm{~ms}$

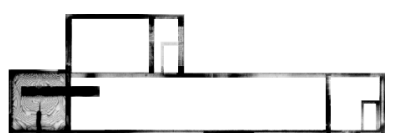

(d) $0.108 \mathrm{~ms}$

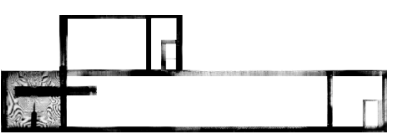

(f) $0.159 \mathrm{~ms}$
Fig. 7 Holographic photograph of the explosion (explosion in room A)

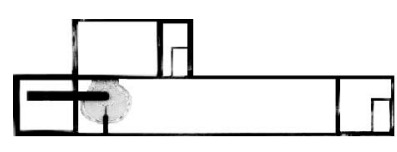

(a) $0.050 \mathrm{~ms}$

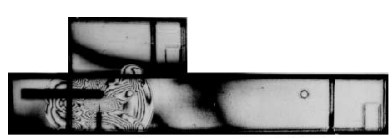

(c) $0.200 \mathrm{~ms}$

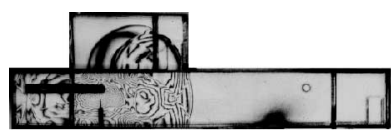

(e) $0.400 \mathrm{~ms}$

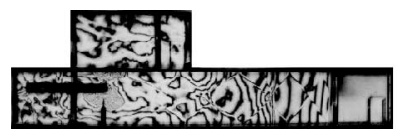

(g) $1.150 \mathrm{~ms}$

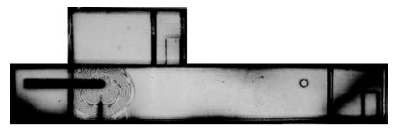

(b) $0.100 \mathrm{~ms}$

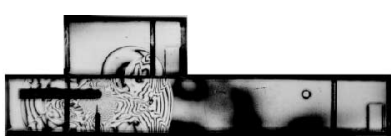

(d) $0.300 \mathrm{~ms}$

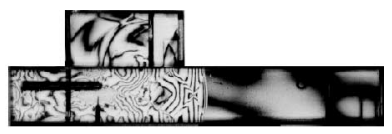

(f) $0.500 \mathrm{~ms}$

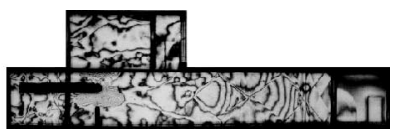

(h) $1.300 \mathrm{~ms}$

Fig. 8 Holographic photograph of the explosion (explosion in room B) (All doors, windows and hatches are opened.)

\section{Numerical Simulation}

\section{1 Simulation model}

We assumed a cubic room (room A) in which the explosion and pressure propagation occurred in order to compare the experimental results with numerical simulation. This model is simulated as the case of the explosion in room A in the visualization experiment. Explosives are assumed as the spherical core of high pressure whose radius is defined as $0.01 \mathrm{~m}$ in room A. All walls are assumed as the slip walls.

An explosive is assumed to be $4.22 \mathrm{~J}$ and the separate window is installed between room A and B. In Fig. 9, an index of 'a' means the length of the separate window. Air temperature is $288 \mathrm{~K}$ uniformly and the pressure is defined as $1 \mathrm{~atm}$ excepting in the area of high pressure.

\subsection{Equations}

Wave propagation and reflection are normally accompanied by dramatic deformation of the latter. Therefore, to investigate these phenomena numerically, the secondorder upwind TVD scheme of Yee's is adopted for these governing equations ${ }^{(5)}$. The basic equations for a threedimensional compressible Eularian flow can be written as follows.

$$
\begin{aligned}
& \frac{\partial \hat{Q}}{\partial t}+\frac{\partial \hat{E}}{\partial \xi}+\frac{\partial \hat{F}}{\partial \eta}+\frac{\partial \hat{G}}{\partial \zeta}=0 \\
& \hat{Q}=\frac{1}{J}\left[\begin{array}{l}
\rho \\
\rho u \\
\rho v \\
\rho w \\
e
\end{array}\right], \hat{E}=\frac{1}{J}\left[\begin{array}{l}
\rho u U+\xi_{x} P \\
\rho v U+\xi_{y} P \\
\rho w U+\xi_{z} P \\
(e+P) U
\end{array}\right], \\
& \hat{F}=\frac{1}{J}\left[\begin{array}{l}
\rho V \\
\rho u V+\eta_{x} P \\
\rho v V+\eta_{y} P \\
\rho w V+\eta_{z} P \\
(e+P) V
\end{array}\right], \hat{F}=\frac{1}{J}\left[\begin{array}{l}
\rho W \\
\rho u W+\zeta_{x} P \\
\rho v W+\zeta_{y} P \\
\rho w W+\zeta_{z} P \\
(e+P) W
\end{array}\right],
\end{aligned}
$$

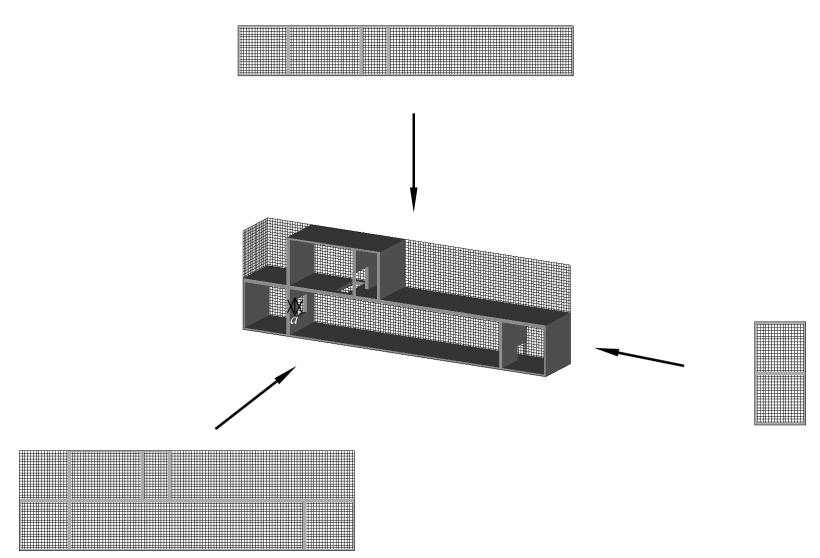

Fig. 9 Grids of numerical simulation 
Here, $\rho$ denotes the density, $u, v$ and $w$ are the velocity components in $x$-, $y$ - and $z$-directions, $e$ is the total energy per unit volume, $p$ is the pressure. And $U, V$ and $W$ mean the $U=\xi_{x} u+\xi_{y} v+\xi_{z} w, V=\eta_{x} u+\eta_{y} v+\eta_{z} w$ and $W=\zeta_{x} u+$ $\zeta_{y} v+\zeta_{z} w$.

\section{Simulation Results}

Figure 10 illustrates the results of numerical simulations. Each figure shows the transient status of pressure wave propagation. Wave expands the spherical shape and it reaches the nearest wall in Fig. 10 (a). A preceding shock wave that reached the floor propagates the sidewalls with occurrence of the reflection in Fig. 10 (b) and (c). In Fig. 10 (c) and (d), wave reaches walls of 2nd floor and ceiling. No directivity of shock wave is observed in Fig. 10 (f). After the reflected wave is slipping along the wall, the wave reaches neighbor wall. After the wave that passes through the window installed between room A and B occurs diffraction at the window, the wave propagates to room B. Since the slipping waves are concentrated at the corner of room A by preceding waves, pressure rise might be observed. And it is also observed that the complex interactions of the reflected waves after the waves reach the wall of axial direction. These figures show the good agreement for experimental results in Fig. 8. This numerical method is suitable to simulate the explosion in scaled model of nuclear fuel cycle facility, because it is sufficient to show the wave propagation with multiple reflections in room $B$ and diffraction phenomena at opened window.

Time dependent pressure tendencies at point A, D and E are shown in Fig. 11. As shown in Fig. 11 (a), point A which is on the axis direction of explosion and it faces on the left-side wall. And Fig. 11 (b) shows the pressure history at the point $\mathrm{D}$ in room $\mathrm{B}$. Two or many pressure peaks are observed simultaneously in each figure. Point $\mathrm{D}$ indicates a corner of the room B. This point locates

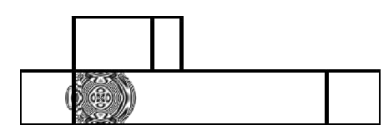

(a) $0.100 \mathrm{~ms}$

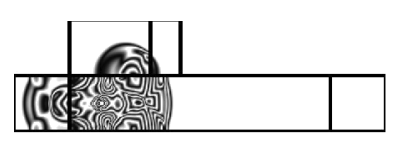

(c) $0.300 \mathrm{~ms}$

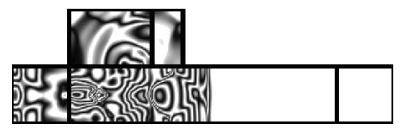

(e) $0.500 \mathrm{~ms}$

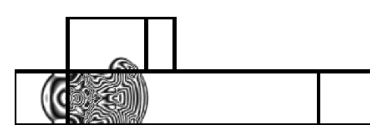

(b) $0.200 \mathrm{~ms}$

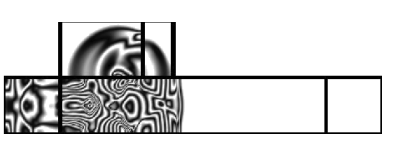

(d) $0.400 \mathrm{~ms}$

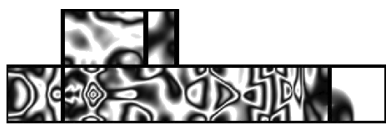

(f) $1.300 \mathrm{~ms}$
Fig. 10 Simulation results (explosion in room B) (All doors, windows and hatches are opened.) the place surrounded in three walls. Pressure waves are thought to gather in this point. It is thought that the first pressure peak is affected by the interaction of the reflected preceding wave, but another pressure peak is also affected by the wave after preceding wave. Then the peak after first shock wave is thought to be caused by waves gathered from three directions. It is concluded that the very large pressure peaks appeared at the corner of the rooms and load to neighbor walls. In comparison with Fig. 11 (a), largest peak pressure in Fig. 11 (c) suggested no attenuation, even the pressure was measured at point $\mathrm{E}$ where it is far from ignition point. And timing of appearance of pressure peaks obtained by numerical simulation correspondents to the timing of them obtained by pressure measurement. Simulation results of peak intensity is almost agreed with results of pressure measurement. But in Fig. 11 (b), measured pressure is quite low, because it is thought that the semiconductor pressure transducer is installed obliquely in point $\mathrm{D}$ for the direction of wave propagation and reflection coefficient of wall of experimental condition is possible to be lower than that of simulation condition.

Time integrated pressure impulse at point D and E is also shown in Fig. 12. Simulation results of time in-

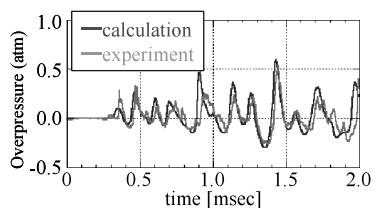

(a) Pressure at point $\mathrm{A}$

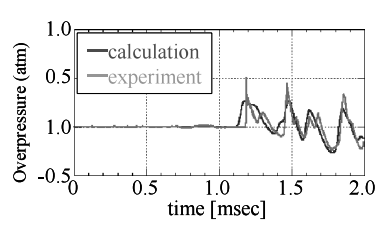

(c) Pressure at point $\mathrm{E}$

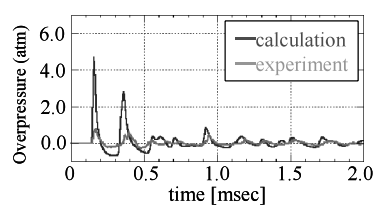

(b) Pressure at point $\mathrm{D}$

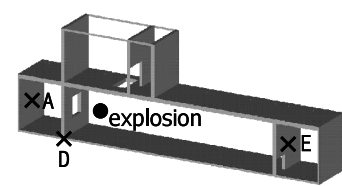

(d) Measurement points
Fig. 11 Time dependent tendency of pressure

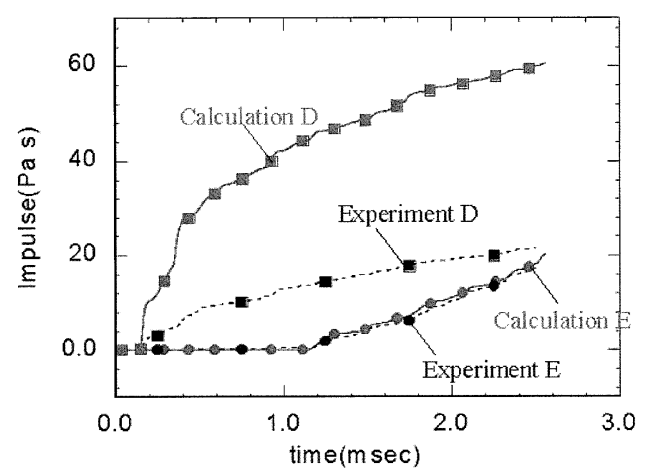

Fig. 12 Impulse of time integrated pressure 
tegrated pressure at point $\mathrm{D}$ is much larger than that of experimental results, because the peak pressure obtained from numerical simulation at point D initially is larger than that obtained from experiment. In Fig. 11, simulation results of peak pressure at point $\mathrm{E}$ is smaller than experimental results. However, both results are agreed in time integrated pressure.

Then the numerical results show the good agreement with experimental result excepting the corners in scaled model, because it is difficult to measure the pressure intensity exactly at the corners.

It is known that the intensity of peak pressure is expanded to explosion energy and peak intensity of highest pressure is tends to fall down along with the window size $^{(6),(7)}$. This large pressure is possible to break the concrete walls of general housings. And it is thought that the effective impact is possible to destroy the facility. Based on these results, in the case of explosion in closed space, it is confirmed that the pressure is extended locally. It can be predicted that large damage might occur at the walls, equipments and so on, when the extended pressure is loaded locally.

\section{Conclusion}

In this study, visualize technique, pressure measurement and numerical methods are adopted for scaled model of nuclear fuel cycle facility. It is confirmed that numerical simulation results agree with the experimental results well and they can explain the phenomena in closed space.

Reflection of the wave is observed repeatedly on the walls, when the explosion occurs in closed space. And it is found that the pressure rise rapidly at the corner of this space. Once wave passes the windows or doors, diffraction occurs around a window and wave propagates in fore direction of walls.

According to these results, it is possible to obtain the following estimation. Sudden pressure rise occurs by the explosion reaches the walls, doors, windows or ceilings.

If the components would notbe strong structure, it is possible for these components to be destroyed by the impact of the pressure (Fig. 12). In case that the pressure wave breaks the components of facility, wave passes through the broken components. Passed wave is driven along the strong walls, corridor or steps, and it attacks another components. But the impact caused by diffracted wave might be small.

Also, it is confirmed that such methods are effective to estimate the damage of facility by explosion and to evaluate safety. It is thought that these technologies will be adopted not only safety assessment of nuclear fuel cycle facilities but also safety design, evaluation for many kinds of facilities.

\section{Acknowledgements}

I would like to thank Dr. Katsumi Tanaka (AIST) and Dr. Atsushi Abe (Tohoku University) for giving us very useful advice and kind helps for this study.

\section{References}

( 1 ) Miura, A., Sato, Y., Koyama, T., Omori, E., Kato, Y., Suzuki, H., Nojiri, I. and Yamanouchi, T., Proceedings of ICONE-9, France, (2001).

( 2 ) U.S. Department of Energy, Tomsk-7 Accident Modeling Summary Report, DOE Official Report, (1996).

( 3 ) U.S. Department of Energy, Accident Investigation Board Report on the May 14, 1997, Chemical Explosion at the Plutonium Reclamation Facility, Hanford Site, Richland, Washington, DOE Official Report (DOE/RL 97-59), (1997).

( 4 ) Takayama, K. and Ben-dor, G., Sadhana, Vol.18, Parts 3 \& 4 (1993), pp.695-710.

( 5 ) Yee, H.C., NASA TM89464, (1987).

(6) Miura, A., Mizukaki, T., Shiraishi, T., Matsuo, A., Takayama, K. and Nojiri, I., Journal of Loss Prevention in the Process Industries, Vol.17 (2004), pp.81-86.

( 7 ) Shiraishi, T., Miura, A., Utsunomiya, G., Abe, A., Matsuo, A., Takayama, K. and Nojiri, I., Proceedings of 2003 Annual Meeting of ASEJL13, (in Japanese), (2003). 